\title{
openheart Economic value of insertable cardiac monitors in unexplained syncope in the United States
}

\author{
Brad S Sutton, ${ }^{1}$ Sarah L Bermingham, ${ }^{2}$ Alexander Diamantopoulos, ${ }^{2}$ \\ Sarah C Rosemas (D) , ${ }^{3}$ Stelios I Tsintzos, ${ }^{4}$ Ying Xia, ${ }^{3}$ Matthew R Reynolds ${ }^{5}$
}

\begin{abstract}
To cite: Sutton BS, Bermingham SL, Diamantopoulos A, et al. Economic value of insertable cardiac monitors in unexplained syncope in the United States. Open Heart 2021;8:e001263. doi:10.1136/ openhrt-2020-001263
\end{abstract}

Received 21 February 2020 Revised 17 September 2020 Accepted 31 January 2021

Check for updates

(C) Author(s) (or their employer(s)) 2021. Re-use permitted under CC BY-NC. No commercial re-use. See rights and permissions. Published by BMJ.

${ }^{1}$ University of Louisville, Louisville, Kentucky, USA ${ }^{2}$ Symmetron Ltd, London, UK ${ }^{3}$ Medtronic Inc, Minneapolis, Minnesota, USA

${ }^{4}$ Health Economics and Reimbursement, Medtronic Europe SA, Tolochenaz, Vaud, Switzerland

${ }^{5}$ Cardiovascular Medicine, Baim Institute for Clinical Research, Boston, Massachusetts, USA

Correspondence to Sarah C Rosemas; sarah.c. rosemas@medtronic.com

\section{ABSTRACT}

Introduction Early use of insertable cardiac monitor (ICM) is recommended for patients with unexplained syncope following initial clinical workup, due to its superior ability to establish symptom-rhythm correlation compared with conventional testing (CONV). However, ICMs incur higher upfront costs, and the impact of additional diagnoses and resulting treatment on downstream costs and outcomes is unclear. We aimed to evaluate the cost-effectiveness of ICM compared with CONV for the diagnosis of arrhythmia in patients with unexplained syncope, from a US payer perspective.

Methods A Markov model was developed to estimate lifetime costs and benefits of arrhythmia diagnosis with ICM versus CONV, considering all related diagnostic and arrhythmia-related treatment costs and consequences. Cohort characteristics and costs were informed by original claims database analyses. Risks of mortality, syncopal recurrence, injury due to syncope and quality of life consequences from syncopal events were identified from the literature.

Results ICM was less costly and more effective than CONV. Most of the observed US\$4532 cost savings were attributed to reduced downstream diagnostic testing. For every 1000 patients, ICM was projected to yield an additional 253 arrhythmia diagnoses and lead to treatment in an additional 168 patients. The ICM strategy resulted in overall improved outcomes ( 0.30 quality-adjusted life years gained), due to a reduction in syncope recurrence and injury resulting from arrhythmia treatment. The results were robust to changes in the base case parameters but sensitive to the model time horizon, underlying probability of syncope recurrence and prevalence of arrhythmias. Conclusions Our model projected that early ICM for the diagnosis of unexplained syncope reduced longterm costs, and led to an improvement in overall clinical outcomes by shortening time to arrhythmia treatment. The cost of ICM was outweighed by savings arising from fewer downstream diagnostic episodes, and the increased cost of treatment was counterbalanced by fewer syncoperelated event costs

\section{INTRODUCTION}

Syncope can be caused by a number of underlying conditions, ranging from benign to life-threatening. ${ }^{1}$ Cardiac causes of syncope

\section{Key questions}

What is already known about this subject?

- Continuous monitoring with insertable cardiac monitors leads to a higher rate of arrhythmia diagnosis in patients with unexplained syncope compared to traditional, opportunistic and single time-point diagnostic testing. The cost-effectiveness of insertable cardiac monitor (ICM) monitoring in the unexplained syncope population is not well understood.

What does this study add?

- In a population of patients with unexplained syncope, the additional diagnostic yield and subsequent arrhythmia therapies received in the ICM arm led to an average gain of 0.30 quality-adjusted life years compared with conventional testing (CONV). Despite a higher initial cost in the ICM strategy, modelling projects long-term overall cost savings, averaging US\$4532 per patient. The cost savings are driven by avoiding repeat diagnostic testing, as well as a reduction in downstream costs related to syncope events and injuries by guiding patients to the appropriate therapy.

How might this impact on clinical practice?

- The use of ICMs to detect cardiac arrhythmias in patients with syncope reduces lifetime costs compared to CONV from a US payer perspective, while enabling better treatment decisions that may improve clinical outcomes in these patients.

include arrhythmias and structural heart disease. Because cardiac syncope can be associated with high mortality in all age groups, ${ }^{2}$ quickly and accurately identifying patients with cardiac causes of syncope is of critical importance.

Clinical guidelines ${ }^{3-5}$ recommend early use of insertable cardiac monitors (ICM) for the evaluation of patients with recurrent, unexplained syncope of suspected arrhythmic cause in whom initial clinical evaluation has not yielded a diagnosis, due to its superior ability to establish symptom-rhythm correlation compared with a conventional testing (CONV) approach. ICMs are inserted 
subcutaneously and continuously scan for arrhythmias. The device automatically records and stores tachycardic or bradycardic events and can also be patient-activated.

Preliminary studies have shown a significant reduction in time to diagnosis and overall costs associated with ICM for the diagnosis of arrhythmia in patients with syncope and no structural heart disease. ${ }^{6-9}$ However, few have evaluated the impact of alternative diagnostic strategies on downstream patient outcomes, and existing clinical codes do not allow direct comparative analysis using claims data. Economic modelling allows the interpretation of test performance using clinically meaningful outcomes by linking the likelihood of ECG capture to the costs and consequences of arrhythmia treatment. ${ }^{10}$

The objective of this study was to evaluate the costeffectiveness of ICM compared with CONV for the diagnosis of arrhythmia in patients with recurrent unexplained syncope in the US. These comparators were evaluated as alternative diagnostic strategies following an inconclusive initial clinical workup; this analysis was not designed to determine the optimal point in the patient pathway to offer ICM technology.

\section{METHODS}

We conducted a cost-utility analysis comparing ICM versus CONV for the diagnosis of unexplained syncope. The analysis was undertaken from a US payer perspective and the model was run over the lifetime of the cohort. Results were expressed in terms of costs, life years, quality-adjusted life years (QALYs), incremental cost per QALY gained, net monetary benefit (NMB), probability of diagnosis, syncope events and syncope-related injuries avoided. All costs were reported in 2018 US dollars (US\$). Costs, QALYs and life years were discounted at the standard annual rate of $3 \% .^{11}$

We reviewed the methodology used in other analyses $^{6-9} 12-14$ to estimate the diagnostic yield, key parameters, assumptions and areas of uncertainty that may impact the cost-effectiveness of syncope diagnosis. We then conducted a targeted literature review to identify published data for each value. An experienced researcher evaluated each identified study according to strength of evidence and potential sources of bias, and whether the population, comparators, outcomes, setting and perspective were within the scope of the model.

We also conducted an analysis of US commercial and Medicare Advantage claims data using the 2008 to 2016 Optum Clinformatics de-identified database to determine the cohort characteristics and healthcare resource use for patients with unexplained syncope. This population included adult ( $\geq 18$ years old) patients with an ICM insertion between 2009 to 2014, with a syncope claim (ICD-9 diagnosis code 780.2: Syncope and collapse) on or within 3 months prior to ICM insertion. Patients with diagnosis codes that could imply causes for transient loss of consciousness on the date of ICM insertion were excluded (eg, anaemia, hypotension, diabetes for hyperglycaemic or hypoglycaemic shock, substance abuse, or neurological conditions).

In a separate analysis, we accessed the most recent available Medicare payment data (2016 calendar year) to determine average payments for hospitalisations for syncope and syncope-related injury. We also used published national average Medicare payment data (2016) to obtain unit costs related to outpatient care and remote monitoring. A mark-up of $25 \%$ was applied to Medicare payments to estimate commercial payments. Final model costs were calculated by applying commercial rates to the proportion of patients with patients in the claims data with commercial plans and those $(66 \%)$ reimbursed by Medicare. All costs were inflated to 2018 US dollars.

\section{Population}

We considered a population of adult patients with unexplained syncope, defined as a recurrent syncopal event with no definite diagnosis following initial clinical workup. The initial evaluation was assumed to include a clinical history and physical examination, 12-lead ECG, basic blood testing and Holter monitoring, as described by the Eastbourne Syncope Assessment Study (EaSyAS)..$^{15}$ Patients with suspected arrhythmia, structural heart disease, pulmonary embolism or other documented potential cause of syncope were not included in this model. Based on baseline characteristics of patients with unexplained syncope identified in the Optum claims analysis, the hypothetical cohort had a median starting age of 73 years, $54 \%$ were women and had experienced an average of $2.3(\mathrm{SD} \pm 4.2)$ syncope events in the year prior to entering the model. Syncope events were defined as a syncope claim (ICD-9 code 780.2 or ICD-10 code R55: Syncope and collapse) occurring in an acute healthcare setting (emergency, urgent care or inpatient facility).

\section{Comparators}

Patients in the model were investigated by either ICM or CONV. Battery life of the device was based on the Reveal LINQ ICM (3 years).

Conventional testing described the default diagnostic pathway when ICM was not used. Diagnostic testing in an outpatient setting was informed by the Optum claims analysis, which found that 12 different diagnostic tests were commonly administered to patients with unexplained syncope (table 1). The average number of tests per syncope was calculated by dividing the frequency of each test by the average number of syncope events experienced in the same period (table 1). CONV in an inpatient setting was based on an analysis of inpatient Medicare claims. After the battery life of ICM expired, patients who experienced a recurrent syncopal event received CONV testing.

\section{Model structure}

The primary goal in the diagnosis of unexplained syncope is to accurately identify the presence or absence 


\begin{tabular}{|c|c|c|c|c|}
\hline Diagnostic test & Reimbursement code & Unit payment* & $\begin{array}{l}\text { Average number of } \\
\text { tests during the year } \\
\text { pre-ICM (baseline } \\
\text { period) }\end{array}$ & $\begin{array}{l}\text { Average number of } \\
\text { tests per syncope } \\
\text { event† }\end{array}$ \\
\hline Emergency department visit & СРТ 99284+APC 5024 & US $\$ 515$ & N/A & 1 \\
\hline Speciality physician consultation & СРТ 99205 & US\$229 & $\mathrm{N} / \mathrm{A}$ & 2 \\
\hline Holter monitor (24-48 hour) & СРТ 93224 & US\$101 & 0.26 & 0.11 \\
\hline External loop recorder & СРТ 93268 & US\$227 & 0.15 & 0.07 \\
\hline Mobile cardiovascular telemetry & СРТ 93229 & US\$805 & 0.17 & 0.07 \\
\hline Extended Holter (up to 21 days) & СРТ 0295T & US\$145 & 0.01 & 0.00 \\
\hline ECG & СРТ 93000 & US\$27 & 5.03 & 2.19 \\
\hline CT (brain) & CРТ 70460+APC 5571 & US\$455 & 1.56 & 0.68 \\
\hline CT (cardiac) & СРТ 75574+APC 5571 & US\$678 & 0.01 & 0.00 \\
\hline MRI (brain) & CPT 70552+APC 5571 & US\$628 & 0.55 & 0.24 \\
\hline MRI (cardiac) & CPT 75559+APC 5523 & US\$409 & 0.02 & 0.01 \\
\hline Exercise test (cardiac stress test) & СРТ 93015 & US\$79 & 0.82 & 0.36 \\
\hline Electroencephalogram & СРТ 95812+APC 5722 & US\$627 & 0.11 & 0.05 \\
\hline Tilt test & СРТ 93660+APC 5723 & US\$659 & 0.16 & 0.07 \\
\hline Electrophysiology study & СРТ 93620+APC 0085 & US\$6468 & 0.12 & 0.05 \\
\hline Coronary angiogram & СРТ 93454+APC 0080 & US\$3979 & 0.20 & 0.09 \\
\hline Carotid Doppler & СРТ 93880+APC 5522 & US\$425 & 0.72 & 0.31 \\
\hline Basic laboratory testing & СРТ 80053 & US\$17 & 4.40 & 1.91 \\
\hline Total weighted average cost & & & US\$14 724 & US\$2536 \\
\hline
\end{tabular}

*Payments are based on national average payments, and represent a weighted average between Medicare and commercial paid amounts based on the proportion of patients with syncope covered by Medicare $(66 \%)$.

†Calculated by dividing the average number of tests in the baseline year by the average number of syncope events (2.3) over the same period.

APC, ambulatory payment classification; CPT, current procedural terminology; CT, computed tomography; ECG, electrocardiogram; ICM, insertable cardiac monitor; MRI, magnetic resonance imaging.

of arrhythmia so that appropriate treatment can be provided.

Our model estimated the probability of experiencing recurrent syncope; injury due to syncope; mortality (not shown); device explant due to battery expiration, adverse event or diagnosis (not shown); and ECG capture and diagnosis according to the underlying cardiac cause of syncope (figure 1A).

A Markov chain was used to model the natural history of patients with syncope (figure 1B). We defined three health states: 'under investigation,' 'on treatment' and 'dead'. Patients with unexplained syncope entered the Markov model under investigation. If arrhythmia was detected, patients transitioned to treatment. Patients could move to the 'dead' state at any point in the model. The cycle length was 1 month.

\section{Baseline transition probabilities and diagnostic yield}

Baseline age-specific and sex-specific all-cause mortality rates were based on the most recent available life tables for the general population, ${ }^{16}$ multiplied by the standardised risk of all-cause mortality in people with cardiac (2.01) and non-cardiac causes of syncope (1.08). ${ }^{2}$
Diagnostic yield was defined as the proportion of patients with syncope recurrence and either an available ECG recording or an automatic arrhythmia detection during the syncopal event. The baseline risk of syncope recurrence ( 0.6 events per year) was based on the rate observed in the EaSyAS study of patients with unexplained syncope after ICM insertion. ${ }^{15}$ The likelihood of capturing an analysable ECG recording during a syncope event was based on the systematic review by Burkowitz et $a l^{17}$ and supplemental targeted search for updated literature in studies with unexplained syncope patients (table 2). The likelihood of ECG capture using a conventional approach was obtained from the only randomised trial reporting this data $\left(\operatorname{EaSyAS}^{15}\right)$. We assumed that the ECG would be interpreted with perfect sensitivity and specificity.

The baseline probability of underlying arrhythmia in patients with unexplained syncope, as well as the proportion of patients with syncope with ventricular arrhythmia, supraventricular arrhythmia and bradycardia, were obtained from a systematic review and meta-analysis of ICM patients with unexplained syncope by Solbiati et al. ${ }^{18}$ 


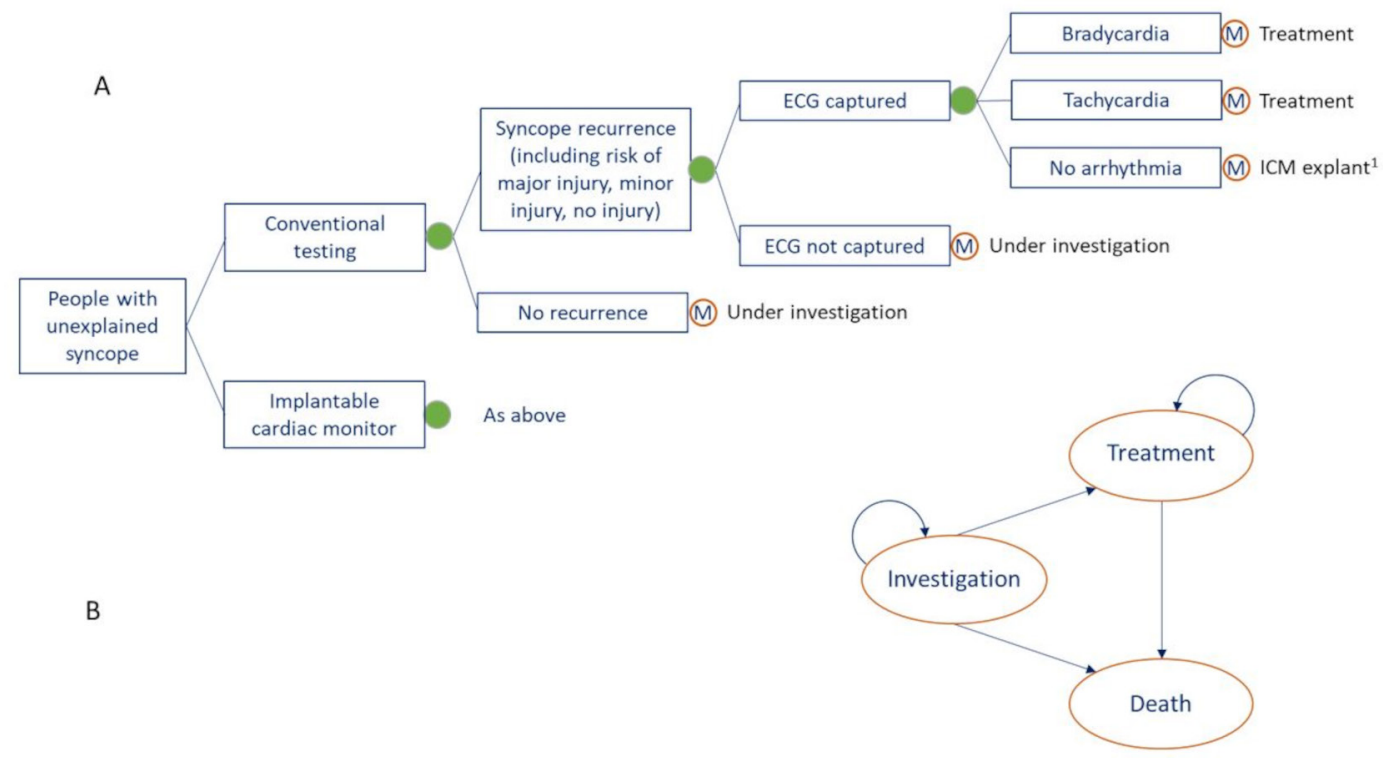

Figure 1 Schematic model structure. 'Circular nodes indicate alternative probability-based outcomes and 'M' nodes indicate entry to the Markov model. Circular arrows in the Markov model indicate residual probabilities (ie, one minus the sum of all other transition probabilities from that health state). ECG, electrocardiogram; ICM, insertable cardiac monitor.

This analysis included 49 studies with 4381 subjects; it was selected to inform the probability of each aetiologic diagnosis as it included all relevant studies published to date and reported values that aligned with our experience in clinical practice. The calculations used to inform the baseline probability of each type of arrhythmia were described in table 2. ICMs must be removed in the case of an adverse event or expired battery; removed devices were assumed not to be replaced. Patients who received a diagnosis of arrhythmia were assumed to have an $80 \%$ probability of ICM removal on diagnosis, compared with $100 \%$ of those discovered to have a normal heart rate and rhythm.

The probability of incurring injury during a syncope event (table 2) was obtained from a study by Bartoletti et $a{ }^{19}{ }^{19}$ the only known study designed to evaluate the incidence and characteristics of both minor and major trauma among patients presenting to the emergency department for syncope. In this study trauma was defined as major when it caused: 'skull or other major bone segments fracture; intracranial haemorrhage; internal organ lesions requiring urgent, specific treatment; retrograde amnesia or focal neurologic defect'. ${ }^{19}$

\section{Costs of testing}

The cost of the ICM device, insertion and removal were based on blended Medicare and commercial payment data (table 3). Based on device monitoring data, $80 \%$ of insertion procedures occurred in an outpatient setting and $20 \%$ inpatient. All explants were outpatient procedures. One month after insertion, patients with ICM had an in-person check-up, and remote monitoring every month thereafter according to the Medicare allowable frequency. Though remote monitoring may occur less frequently, conservative cost savings were assumed.
The cost of conventional testing in an outpatient setting was calculated by weighting blended reimbursement unit costs by the frequency of testing obtained from Optum claims analysis, plus the cost of the emergency visit and two specialist consultations based on clinical experience (table 1). The cost of CONV syncope evaluation in an inpatient setting was calculated based on the blended payer payment for a hospitalization with DRG 312 (Syncope and collapse) with no diagnosis code for physical injuries or trauma. The final cost of CONV assumed that $30 \%$ of patients received conventional testing in an outpatient setting.

Initial diagnostic work-up occurring prior to model entry was common to all patients, and hence was not included in the cost of either comparator.

\section{Costs and consequences of treatment}

Patients with confirmed bradycardia received a pacemaker. The cost of pacemaker implantation was obtained from national average Medicare payment data (table 3). Monthly pacemaker monitoring costs were calculated per Medicare reimbursement allowable frequencies. Pacemaker treatment for bradycardia resulted in a $90 \%$ reduction in syncope recurrence, as reported by the International Study on Syncope of Uncertain Etiology 2 (ISSUE 2) ${ }^{20}$ In the absence of robust evidence and based on clinical experience, it was assumed that bradycardia patients with a pacemaker had the same mortality risk as the general population.

We assumed $95 \%$ of patients with ventricular tachycardia and ventricular fibrillation $(\mathrm{VT} / \mathrm{VF})$ were indicated for an implantable cardioverter-defibrillator (ICD) and the rest for ablation. The cost of ICD implantation and ablation were obtained from national average Medicare payment data (table 3 ). Monthly ICD monitoring 
Arrhythmias and sudden death

Table 2 Baseline probabilities and diagnostic accuracy of conventional testing versus ICM for unexplained syncope

\begin{tabular}{|c|c|c|}
\hline Parameter & Mean & Source \\
\hline \multicolumn{3}{|l|}{ Mortality } \\
\hline Monthly probability for general population & Age and sex-specific & US Centers for Disease Control ${ }^{16}$ \\
\hline HR for cardiac vs no syncope & 2.01 & Soteriades et al $2002^{2}$ \\
\hline HR for vasovagal vs no syncope & 1.08 & Soteriades et al $2002^{2}$ \\
\hline \multicolumn{3}{|l|}{ Cardiac cause of syncope } \\
\hline Proportion of unexplained syncope patients with arrhythmia & $60.4 \%$ & \multirow{4}{*}{$\begin{array}{l}\text { Solbiati et al, } 2017^{18} \text {; meta-analysis of } 4381 \\
\text { patients }\end{array}$} \\
\hline Proportion of arrhythmia patients with bradycardia* & $68.7 \%$ & \\
\hline $\begin{array}{l}\text { Proportion of arrhythmia patients with ventricular tachycardia } \\
\text { and ventricular fibrillation* }\end{array}$ & $10.2 \%$ & \\
\hline $\begin{array}{l}\text { Proportion of arrhythmia patients with supraventricular } \\
\text { tachycardia or atrial fibrillation* }\end{array}$ & $21.1 \%$ & \\
\hline
\end{tabular}

\begin{tabular}{|c|c|c|}
\hline \multicolumn{3}{|l|}{ Syncope recurrence } \\
\hline Monthly probability of syncope recurrence & $5.1 \%$ & $\begin{array}{l}\text { EaSyAS }^{15} \text {; monthly risk corresponding to event } \\
\text { rate of } 0.6 \text { events/year }\end{array}$ \\
\hline \multicolumn{3}{|l|}{ Injury due to syncope } \\
\hline Probability of major injury per syncope event & $4.8 \%$ & Bartoletti et al, $2008^{19}$ \\
\hline Probability of minor injury per syncope event & $24.7 \%$ & Bartoletti et al, $2008^{19}$ \\
\hline \multicolumn{3}{|l|}{ Probability of ECG capture } \\
\hline Conventional testing & $18.9 \%$ & Farwell et al, $2006^{43}$ \\
\hline Reveal LINQ ICM & $\begin{array}{l}\text { Month } 0=70 \% \\
\text { Month } 1=82 \% \\
\text { Month } 2=78 \% \\
\text { Month } 3=87 \% \\
\text { Month } 4=90 \% \\
\text { Month } 5 \text { onward=97\% }\end{array}$ & Musat et al, $2017^{44}$ \\
\hline
\end{tabular}

\begin{tabular}{lll}
\hline Battery life & 3.0 years & \\
\hline Reveal LINQ ICM & & \\
\hline Adverse events & $\begin{array}{l}\text { First cycle }=0.00734 \\
\text { Subsequent cycles }=0\end{array}$ & $\begin{array}{l}\text { Pooled data from LINQ ICM usability and registry } \\
\text { studies }\end{array}$ \\
\hline Risk of AE requiring ICM explant & & Assumption \\
\hline Probability of ICM removal (explant) & $80 \%$ & Assumption \\
\hline Probability of removal on diagnosis of arrhythmic syncope & $100 \%$ & \\
\hline Probability of removal on diagnosis of non-arrhythmic syncope & & \\
\hline
\end{tabular}

*See online supplemental files for a breakdown of how these probabilities were calculated.

AE, adverse event; EaSyAS, Eastbourne Syncope Assessment Study; ECG, electrocardiogram; HR, hazard ratio; ICM, insertable cardiac monitor; US, United States.

costs were calculated per Medicare allowable frequencies. Based on clinical experience, ICD and ablation therapy for patients with $\mathrm{VT} / \mathrm{VF}$ resulted in a $30 \%$ reduction in syncope recurrence. Treatment for $\mathrm{VT} / \mathrm{VF}$ reduced mortality risk by $33 \%$ compared with untreated patients, as reported by the Antiarrhythmics Versus Implantable Defibrillators (AVID) trial. ${ }^{21}$

We assumed $75 \%$ of patients with supraventricular tachycardia and atrial fibrillation (SVT/AF) were indicated for ablation and the rest for medication. The method used to determine the cost of anticoagulant, rate and rhythm controlling drugs is described in the online supplemental files. Patients treated for SVT/AF benefited from a $95 \%$ reduction in recurrent syncope based on a systematic review by Spector et $a .^{22}$ In the absence of evidence, it was assumed that ablation and medication for SVT/AF had no impact on mortality.

\section{Health state utilities}

A multiplicative method was used to estimate healthstate utility values for patients with unexplained syncope, recurrent syncope and injury due to syncope (table 4). Average age-stratified EuroQol 5-dimension (EQ-5D) scores from the US general population were used to inform baseline utility.

There are no EQ-5D values available to describe the utility of patients with unexplained syncope. Instead, SF-36 domain scores reported by van Dijk et $a t^{23}$ for 
Table 3 Unit costs

\begin{tabular}{|c|c|c|c|c|}
\hline Description & Reimbursement code & $\begin{array}{l}\text { Medicare } \\
\text { payment }\end{array}$ & $\begin{array}{l}\text { Commercial } \\
\text { payment }\end{array}$ & $\begin{array}{l}\text { Blended } \\
\text { payment* }^{\star} \\
\text { (2018 US\$) }\end{array}$ \\
\hline ICM device and insertion & $\begin{array}{l}\text { Weighted average of: } \\
80 \% \text { outpatient (APC } 0680 \text { plus physician fees CPT } \\
33282 \text { : } \$ 7812 \text { blended payment*), and } 20 \% \text { mean } \\
\text { inpatient admission (DRG } 260,261 \text {, or } 262 \text { in patients } \\
\text { with ICD-10 0JH602Z or 0JH632Z or CPT } 33282: \$ 18628 \\
\text { blended) }\end{array}$ & US\$9209 & US\$11 511 & US\$9975 \\
\hline ICM explant & $\begin{array}{l}\text { Total outpatient (APC 0020) plus physician fees (CPT } \\
\text { 33284) }\end{array}$ & US\$746.00 & US\$932.50 & US\$808.10 \\
\hline Monthly ICM monitoring & $\begin{array}{l}\text { Monthly average of one in-person check } 1 \text { month after } \\
\text { insertion (CPT 93291) and remote checks every month } \\
\text { thereafter (CPT 93298+CPT 93299) }\end{array}$ & US\$72.18 & US $\$ 90.22$ & US\$78.18 \\
\hline Conventional testing & $\begin{array}{l}\text { Weighted average of } 70 \% \text { inpatient admission (DRG } \\
312 \text { with no ICD code for injuries: US } \$ 8296 \text { blended } \\
\text { payment*) and } 30 \% \text { outpatient testing: US\$2536 blended } \\
\text { (table 1) }\end{array}$ & US\$6063 & US\$7579 & US\$6568 \\
\hline Cost of medication per month & See online supplemental files & NA & NA & US\$640 \\
\hline $\begin{array}{l}\text { Pacemaker device and } \\
\text { implantation }\end{array}$ & $\begin{array}{l}\text { Total outpatient (APC 0655) plus physician fees (CPT } \\
\text { 33208) }\end{array}$ & US\$9958 & US\$12 448 & US\$10 852 \\
\hline Ablation & $\begin{array}{l}\text { Total outpatient (APC 8000) plus physician fees (CPT } \\
\text { 93656) }\end{array}$ & US\$19 693 & US\$24 616 & US\$21 332 \\
\hline $\begin{array}{l}\text { Defibrillator device and } \\
\text { implantation }\end{array}$ & $\begin{array}{l}\text { Total outpatient (APC 0108) plus physician fees (CPT } \\
33249 \text { ) }\end{array}$ & US\$31 639 & US\$39 549 & US\$34 273 \\
\hline Monthly pacemaker follow-up & CPT $93280+1$ outpatient consultation every 6 months & US\$72 & US\$90 & US\$78 \\
\hline Monthly defibrillator follow-up & $\begin{array}{l}\text { CPT 93289+2 in-person and three remote consultations } \\
\text { in the first year, then one in-person and three remote } \\
\text { consultations every year after }\end{array}$ & & & \\
\hline Monthly medication follow-up & CPT 93280+1 outpatient consultation every 6 months & US\$72 & US\$90 & US\$78 \\
\hline Major injury & $\begin{array}{l}\text { Mean cost of hospitalisation for any injury-related DRG } \\
\text { plus ICD code for syncope (ICD-10 R55 Syncope or ICD- } \\
10195.1 \text { Orthostatic Hypotension). Top and bottom } 1 \% \text { of } \\
\text { data were trimmed to remove outliers. The average cost } \\
\text { across the }>85^{\text {th }} \text { percentile were assumed to represent } \\
\text { major injury based on the occurrence of major trauma in } \\
\text { Bartoletti et al } 2008 .{ }^{19}\end{array}$ & US\$31742 & US\$39 678 & US\$34 385 \\
\hline Minor injury & $\begin{array}{l}\text { Mean cost of hospitalisation for any injury DRG plus } \\
\text { ICD code for syncope (ICD-10 R55 Syncope or ICD- } 10 \\
\text { I95.1 Orthostatic Hypotension). Top and bottom } 1 \% \text { of } \\
\text { data were trimmed to remove outliers. The average cost } \\
\text { across the } \leq 85^{\text {th }} \text { percentile were assumed to represent } \\
\text { minor injury based on the occurrence of minor trauma in } \\
\text { Bartoletti et al } 2008 .^{19}\end{array}$ & US\$9409 & US\$11 761 & US\$10 192 \\
\hline
\end{tabular}

*Payments are based on national average payments, and represent a weighted average between Medicare and commercial paid amounts based on the proportion of patients with syncope covered by Medicare (66\%).

APC, ambulatory payment classification; CPT, current procedural terminology; DRG, diagnosis-related group; ICD-10, 10th Revision of the International Classification of Diseases; ICM, insertable cardiac monitor; USD, US dollar.

patients with syncope compared with matched controls from the Dutch general population were mapped to EQ-5D values using an algorithm established by Ara and Brazier. ${ }^{24}$ The percentage difference in health utility between patients with syncope and the general population observed by van Dijk et $a l^{23}$ was multiplied by the average health utility of the US population to obtain an estimate of utility for patients with unexplained syncope.

Syncope recurrence can negatively affect quality of life. ${ }^{23}$ 25-27 To calculate the utility decrement due to syncope recurrence, we mapped SF-36 scores to EQ-5D utilities for those with and without syncope recurrence 
Table 4 Mapped health state utilities

\begin{tabular}{|c|c|c|c|c|c|c|c|c|c|c|c|c|}
\hline \multirow[b]{2}{*}{ Population } & \multirow[b]{2}{*}{$\mathbf{N}$} & \multicolumn{8}{|c|}{ SF-36 mean dimension score } & \multirow{2}{*}{$\begin{array}{l}\text { Mapped } \\
\text { EQ-5D* }\end{array}$} & \multirow{2}{*}{$\begin{array}{l}\text { Mapped EQ- } \\
5 D \text { as a } \% \text { of } \\
\text { reference value }\end{array}$} & \multirow[b]{2}{*}{ Source } \\
\hline & & PF & $\mathbf{R P}$ & BP & GH & SF & RE & MH & $\mathbf{V}$ & & & \\
\hline \multicolumn{12}{|c|}{ General population } & \multirow{6}{*}{$\begin{array}{l}\text { van Dijk et al, } \\
2006^{23}\end{array}$} \\
\hline Male & 976 & 85 & 79 & 77 & 72 & 86 & 86 & 79 & 72 & 0.859 & $\mathrm{~N} / \mathrm{A}$; baseline & \\
\hline Female & 766 & 80 & 74 & 72 & 70 & 82 & 79 & 74 & 64 & 0.812 & $\mathrm{~N} / \mathrm{A}$; baseline & \\
\hline \multicolumn{12}{|c|}{ People with unexplained syncope } & \\
\hline Male & 222 & 70 & 47 & 67 & 56 & 69 & 60 & 67 & 52 & 0.731 & $85 \%$ & \\
\hline Female & 163 & 64 & 39 & 60 & 52 & 66 & 62 & 64 & 44 & 0.683 & $84 \%$ & \\
\hline \multicolumn{10}{|c|}{ People with syncope recurrence vs non-recurrence } & & & $\begin{array}{l}\text { Barón-Esquivias } \\
\text { et al, } 2005^{25}\end{array}$ \\
\hline $\begin{array}{l}\text { With } \\
\text { recurrence }\end{array}$ & 33 & 85 & 100 & 61 & 57 & 87 & 66 & 56 & 45 & 0.755 & $85 \%$ & \\
\hline $\begin{array}{l}\text { Without } \\
\text { recurrence }\end{array}$ & 134 & 90 & 100 & 79 & 72 & 100 & 100 & 72 & 60 & 0.886 & Reference & \\
\hline Baseline & 167 & 90 & 100 & 72 & 62 & 88 & 100 & 68 & 65 & 0.834 & NA; baseline & \\
\hline \multicolumn{12}{|c|}{ Older people with limitations vs no limitations in activities of daily living resulting from falling } & $\begin{array}{l}\text { Rodrigues et al, } \\
2013^{29}\end{array}$ \\
\hline $\begin{array}{l}\text { Not suffered } \\
\text { a fall }\end{array}$ & 1340 & 76 & 80 & 77 & 72 & 85 & 88 & 78 & 73 & 0.822 & N/A; baseline & \\
\hline $\begin{array}{l}\text { With } \\
\text { limitations }\end{array}$ & 52 & 55 & 58 & 60 & 67 & 67 & 72 & 71 & 63 & 0.660 & $80 \%$ & \\
\hline $\begin{array}{l}\text { Without } \\
\text { limitations }\end{array}$ & 39 & 72 & 78 & 70 & 74 & 80 & 88 & 72 & 67 & 0.769 & $94 \%$ & \\
\hline
\end{tabular}

*Mapped using the algorithm described by Ara and Brazier 2008. ${ }^{24}$

$\mathrm{BP}$, bodily pain; $\mathrm{GH}$, general health; $\mathrm{MH}$, mental health; NA, not applicable; PF, physical functioning; RE, role emotional; RP, role physical; SF, social functioning; V, vitality.

reported by Barón-Esquivias et al. ${ }^{25}$ The proportional difference in mapped values for people with recurrent syncope compared with non-recurrent syncope was applied to the utility of people with unexplained syncope to obtain the utility decrement for people with recurrent syncope. This impact on quality of life was reported to improve within 6 months when the frequency of syncope was reduced by permanent pacemaker implantation. ${ }^{28}$ Therefore, we assumed the decline in quality of life would affect patients for 6 months following recurrence.

Because utility values for patients with injury due to syncope were not identified, a proxy was used. SF-36 domain scores reported by Rodrigues ${ }^{29}$ for patients with and without fall-related limitations were mapped to EQ-5D values ${ }^{24}$. The proportional decrease in mapped
EQ-5D values for people who experienced limitations compared with no limitations because of falling was applied for 6 months to the utility of people with syncope recurrence to obtain the utility of people with major and minor trauma due to syncope.

\section{RESULTS \\ Base case}

The base case analysis found that use of an ICM strategy was less costly over a lifetime horizon (with a savings of $\$ 4,532$ ), and on average led to 0.30 additional QALYs compared with CONV due to higher rates of diagnosis and therapeutic intervention for arrhythmias (table 5).

\section{Table 5 Base case results for CONV and ICM}

\begin{tabular}{lllllll}
\hline Comparator & Total costs & Total QALYs & $\Delta$ costs & D QALYs & ICER & $\begin{array}{l}\text { NMB } \\
\text { (WTP=US\$100 000) }\end{array}$ \\
\hline CONV & US\$41644 & 5.7307 & Baseline & Baseline & Baseline & US\$531424 \\
ICM & US $\$ 37111$ & 6.0313 & US\$4532 & -0.3007 & Dominates & US\$566 021 \\
\hline
\end{tabular}

${ }^{*}$ Net monetary benefit (NMB) is a summary statistic that represents the value of an intervention in monetary terms given the willingnessto-pay per unit of benefit (for example, a QALY). NMB is calculated as: (benefit $x$ willingness to pay threshold) - cost. A larger NMB value indicates greater cost-effectiveness of the intervention.

CONV, conventional testing; ICER, incremental cost-effectiveness ratio; ICM, insertable cardiac monitor; NMB, net monetary benefit; QALY, quality-adjusted life years; WTP, willingness-to-pay threshold. 


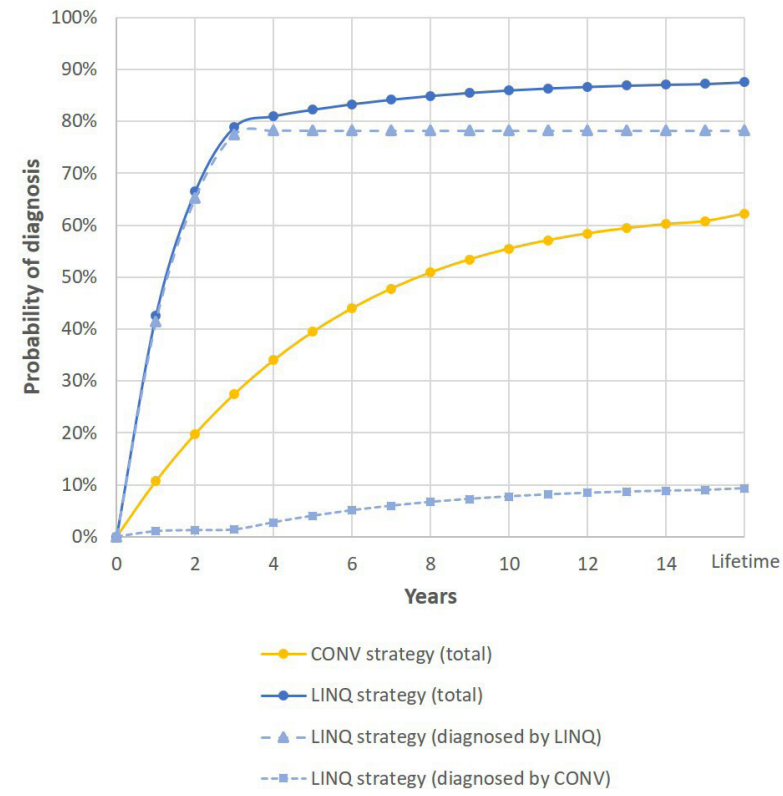

Figure 2 Probability of diagnosis over time. CONV,conventional testing.

Patients received a faster diagnosis with ICM compared with the CONV strategy; a total of $87.5 \%$ patients in the ICM group received a positive or negative (rule-out) diagnosis, compared with $62.2 \%$ in the CONV group. In the ICM group, $90 \%$ of diagnoses occurred by end of year 3 , whereas this outcome took 11 years for patients in the CONV group (figure 2). Consequently, patients with ICM experienced fewer downstream diagnostic tests and received appropriate treatment sooner than those in the CONV arm.

For every 1000 patients, ICM was found to yield an additional 253 arrhythmia diagnoses, leading to treatment in an additional 168 patients. Over a lifetime horizon the model predicts 896 fewer syncopal events and 264 fewer syncope-related injuries would be incurred compared with CONV, as a result of additional therapeutic intervention. Patients diagnosed with ICM experienced an average of 4.3 recurrent syncope events over their lifetime, compared with 5.2 for patients diagnosed with CONV.

Most cost savings associated with ICM were due to reduced total diagnostic costs $(\$ 14,813$ vs. $\$ 19,007$, figure 3). Although a higher probability of diagnosis led to increased treatment costs for patients with ICM, this was balanced by decreased costs of investigating syncope recurrences in yet undiagnosed patients, and treating syncope-related injury (figure 3).

Considering the overall likelihood of diagnosis (arrhythmia or rule-out) and the total diagnostic costs, the average cost-per-diagnosis was approximately 1.8 times higher in the CONV strategy at US\$30 558 compared to US\$16 929 with ICM.

The correct identification and treatment of patients for pacing, ablation, medication and ICD therapy resulted in more QALYs using an ICM strategy compared with CONV (table 5). This improvement was due to a reduction in projected recurrent syncope events and associated trauma as a result of the successful diagnosis and treatment of underlying arrhythmia, and to a lesser degree due to reduced mortality risks with accelerated ICD treatment for $\mathrm{VT} / \mathrm{VF}$.

\section{Sensitivity analysis}

One-way sensitivity analyses were undertaken to test the robustness of the model. The ranges used to explore the impact of each parameter were based on upper and lower estimates in the literature or by halving and doubling the base case values where published estimates were not available.

Deterministic sensitivity analyses found that the conclusions of the analysis were robust to changes in most variables (figure 4 ). The model was most sensitive to a decreased probability of recurrent syncope. When the monthly probability of recurrent syncope was assumed to be equal to the value $(3.6 \%)$ reported in the PICTURE (Place of Reveal In the Care pathway and Treatment of

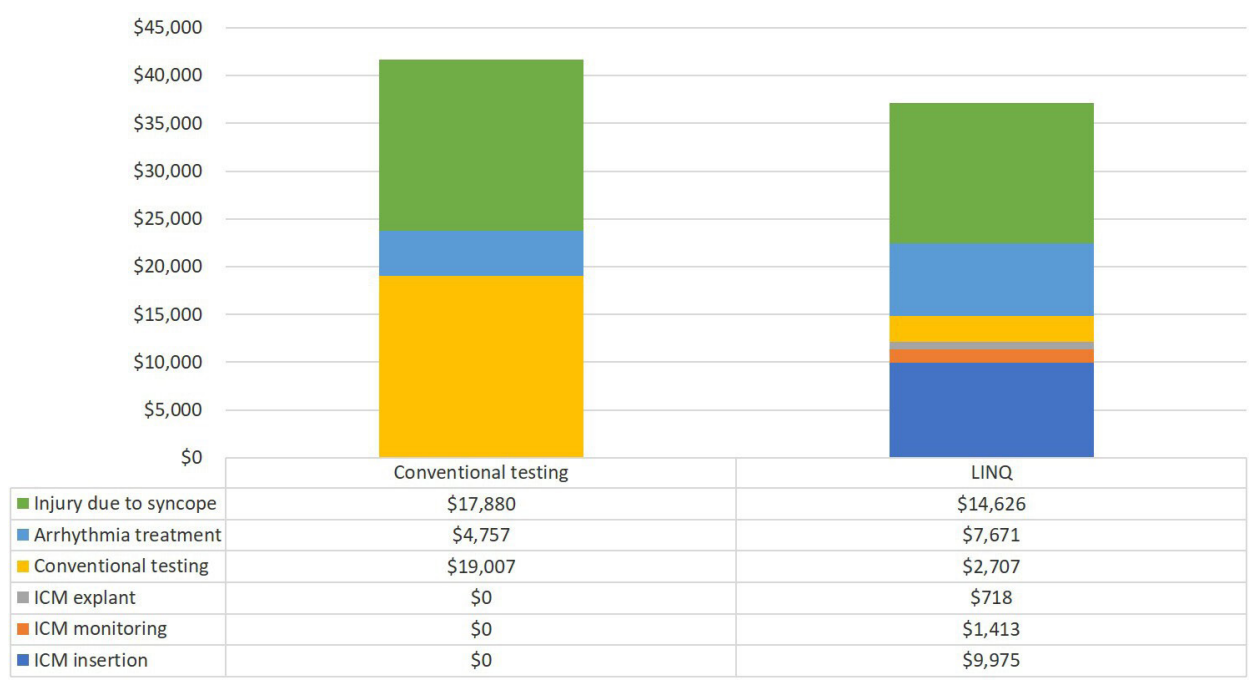

Figure 3 Breakdown of total costs for conventional testing and ICM. ICM,insertable cardiac monitor. 
Incremental Cost per QALY Gained (USD)

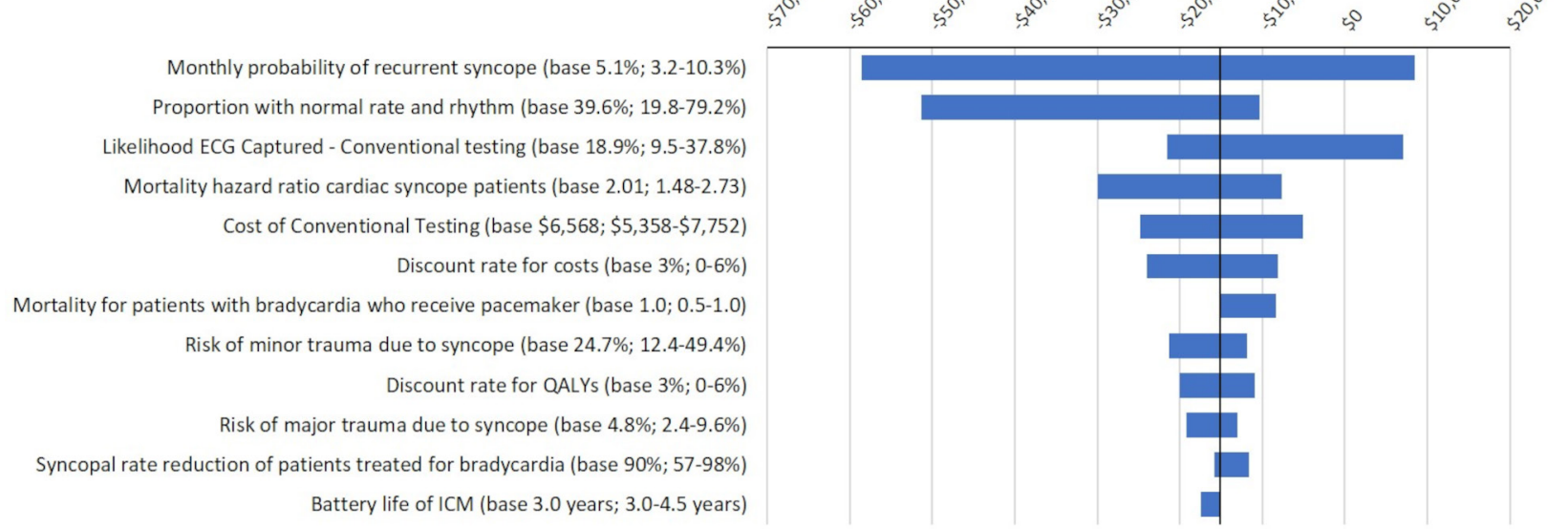

Figure 4 Tornado diagram of one-way sensitivity analyses. The 12 most influential variables are shown here; please refer to online supplemental files for a tornado diagram with all one-way sensitivity analyses. ECG, electrocardiogram; ICM,insertable cardiac monitor; QALY, quality-adjusted life years; USD, US dollar.

patients with Unexplained Recurrent Syncope) registry, ${ }^{30}$ ICM remained highly cost-effective with an incremental cost-effectiveness ratio (ICER) of US $\$ 2255$ per QALY. Threshold analyses found that when the monthly probability of syncope was reduced to $<1.4 \%$ (one quarter of the base case estimate), the ICER exceeded US\$50 000 per QALY gained.

The results of the model were also influenced by the underlying prevalence of arrhythmia. The lower the probability of cardiac syncope, the less cost-effective the ICM strategy would become. However, the conclusions of the model were robust to alternative rates in the probability of underlying arrhythmia reported in the literature. ${ }^{4}$ In extreme value testing, even when the likelihood that syncope was caused by arrhythmia was reduced by $50 \%$, ICM remained a more effective and cost-saving strategy.

Lengthening the assumed ICM battery longevity from 3 to 4.5 years increased the projected cost savings of ICMs from US\$4 532 to US\$5 736 (an additional US $\$ 1204$ savings), and led to an additional 0.03 QALYs. This was driven by an increase in the syncope diagnosis and treatment rates, with an additional 28 estimated diagnoses per 1000 patients, and consequent decrease in the syncope events incurred (78 fewer syncopal events per 1000 patients).

The cost-effectiveness of ICM was sensitive to the time horizon of the analysis. The model was sensitive to a time horizon of $<10$ years; ICM was found to be more costly than CONV on a time horizon of $\leq 5$ years (figure 5 ).

\section{DISCUSSION}

We developed a Markov cohort decision analytical model to compare the cost-effectiveness of ICM versus CONV for the diagnosis of unexplained syncope. Our model predicted that ICM is more effective and cost saving

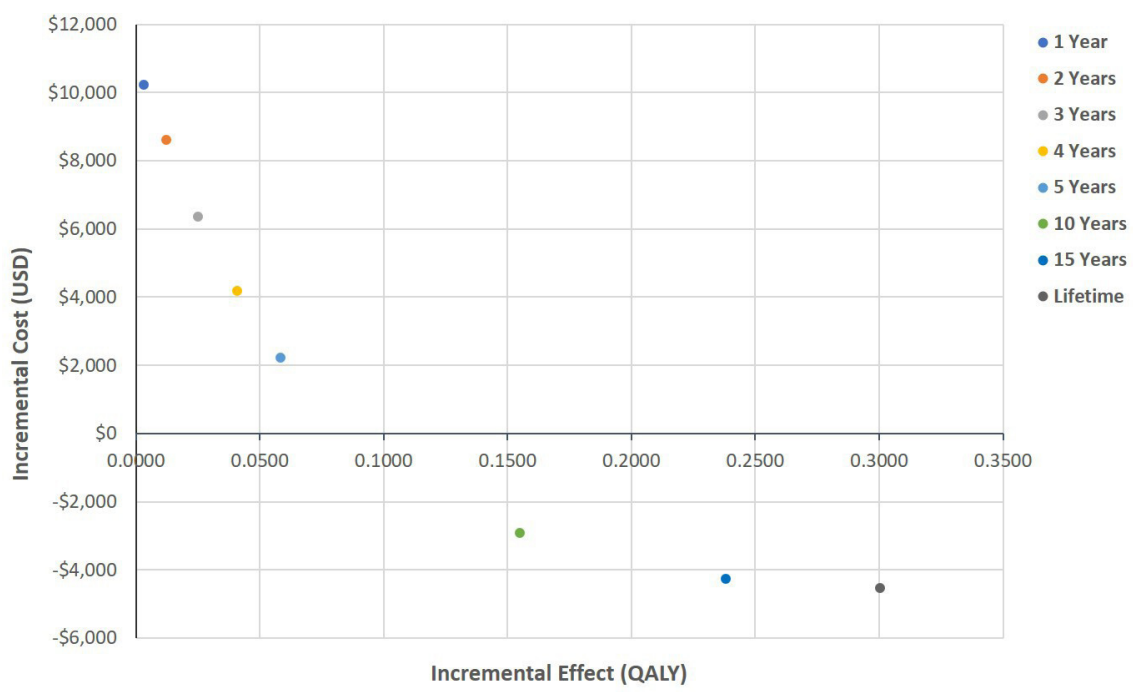

Figure 5 The cost-effectiveness of ICM compared with conventional testing over alternative time horizons. ICM,insertable cardiac monitor; QALY, quality-adjustedlife years; USD, US dollar. 
compared with CONV in patients with unexplained syncope. By quickly guiding patients to appropriate therapy, patients who received ICM were expected to have fewer repeat diagnostic tests, recurrent syncope events and syncope-related injuries than those who received CONV. Our conclusion was consistent across the extreme ranges of most model parameters, except for the model time horizon and the risk of recurrent syncope.

The timely and accurate diagnosis of cardiac syncope has implications for the treatment and management decisions made on behalf of the patient, which can have longlasting consequences as far as subsequent health risks, costs and quality of life that persist for the remainder of a person's life. In such instances, a lifetime time horizon is appropriate for the base case analysis. ${ }^{31-33}$ However, because clinical decision-makers sometimes adopt a shorter perspective, the cost-effectiveness of ICM was evaluated over shorter horizons in sensitivity analyses. Although ICM has an ICER that would be considered cost-effective in most jurisdictions over all time horizons, cost savings are only accrued over a time horizon of more than 5 years. This analysis found that ICM was cost-effective across the range of recurrent syncope probabilities reported in the literature $(3.2 \%$ to $10.3 \%$ per month) $)^{3034-40}$ at a threshold of US\$50 000 per QALY gained.

This study builds on two previous cost-effectiveness analyses $^{79}$ and one financial impact model ${ }^{8}$ that compared ICM with CONV for the diagnosis of arrhythmia in unexplained syncope. When we explored the impact of a shorter battery life, our results were consistent with the finding by Rogers $e t a t^{\vartheta}$ that the earliest generation of the Reveal ICM was associated with an ICER of approximately US $\$ 14000$. The higher ICER reported by Davis $e t a l^{7}$ can be explained by their omission of downstream reduction in mortality, syncope recurrence or injury.

Our study was the first to evaluate the cost-effectiveness of the Reveal LINQ ICM. A key strength of our model was the addition of original data obtained from a large, nationally representative claims database to inform baseline population characteristics, resource use and cost estimates. In addition, whereas previous models assumed all patients with arrhythmia received either pacemakers or ICD, our model captured all treatment pathways, in addition to potential recurrent syncope events and risk of syncope-related injury. Therefore, our model represents the most comprehensive estimate of the costs and consequences of the diagnosis of unexplained syncope to date.

Evidence gathered directly in clinical trials or from analysing claims databases should in theory have been available by now and able to answer our research question. However, the lack of appropriate coding for unexplained syncope and requirement for long-term continuous enrolment prohibits these analyses in claims. The modelling approach remains the only reliable method to predict those outcomes while accounting for uncertainty.
Several limitations which were explored in sensitivity analysis. First, there was limited evidence regarding the ability of pacemakers, ICD, ablation and medication to reduce syncope recurrence and overall mortality in patients with arrhythmia. The main driver of increased QALYs for patients with ICM was predominately the utility benefit attributed to a reduction in recurrent syncope events. Increased QALYs resulting from avoidance of injuries and a reduction in mortality attributed to ICD placement for VT/VF did not impact the conclusion of the analysis. Second, in the absence of preference-based utility values derived from people with syncope, we estimated utilities using a multiplicative method. Although considered the most methodologically sound approach to calculating utility values where none exist in the literature ${ }^{41}$ these values should be interpreted with caution. Third, we assumed the sensitivity and specificity of a physician-read ECG was $100 \%$. However, as demonstrated by the SAFE trial, ${ }^{42}$ non-cardiac specialists have lower sensitivity and specificity than a specialist determination. Because ICMs are usually implanted in centres staffed by specialists, it is doubtful that this assumption would change the results of the analysis but is worth considering before implementing ICM in settings where the ECG is unlikely to be interpreted by a specialist. Two questions were considered outside the scope of this analysis. First, we did not consider a strategy of delayed ICM to be a relevant comparator. Second, the model did not consider whether to replace the ICM after battery expiration.

Our model projected that early ICM for the diagnosis of unexplained syncope reduced long-term total costs, and led to increased QALYs by shortening the time to arrhythmia treatment. The cost of ICM was outweighed by savings arising from fewer downstream diagnostic episodes, and the increased cost of treatment was counterbalanced by fewer syncope-related events. Future research should seek to investigate the probability of recurrent syncope, methods to determine the underlying risk of arrhythmia and long-term outcomes of patients with unexplained syncope. More research is also needed into potential benefits of ICM diagnosis that were not captured by our model, such as a potential improvement in health utility resulting from an early diagnosis of nonarrhythmic causes of syncope.

Contributors $S B, A D, S R, S T$ and $Y X$ conceived the analysis. $S B, A D$ and $Y X$ conducted the analysis. BS and MR validated the model structure, assumptions and inputs. SB, SR and ST drafted the initial version of the manuscript. All authors provided critical feedback on the manuscript and have approved the final version.

Competing interests SR and AD are employed by Symmetron Ltd, which received funding from Medtronic plc for this analysis. BS and MR have received consultancy fees from Medtronic plc. SR, ST and YX are employees and have equity interest in Medtronic plc.

Patient consent for publication Not required.

Provenance and peer review Not commissioned; externally peer reviewed.

Data availability statement All data relevant to the study are included in the article or uploaded as supplementary information.

Open access This is an open access article distributed in accordance with the Creative Commons Attribution Non Commercial (CC BY-NC 4.0) license, which 
permits others to distribute, remix, adapt, build upon this work non-commercially, and license their derivative works on different terms, provided the original work is properly cited, appropriate credit is given, any changes made indicated, and the use is non-commercial. See: http://creativecommons.org/licenses/by-nc/4.0/.

ORCID iD

Sarah C Rosemas http://orcid.org/0000-0001-8864-2734

\section{REFERENCES}

1 da Silva RMFL. Syncope: epidemiology, etiology, and prognosis. Front Physiol 2014:5:1-4.

2 Soteriades ES, Evans JC, Larson MG, et al. Incidence and prognosis of syncope. N Engl J Med 2002;347:878-85.

3 Brignole M, Moya A, de Lange FJ, et al. 2018 ESC guidelines for the diagnosis and management of syncope. Eur Heart $J$ 2018;39:1883-948.

4 National Institute of Health and Care Excellence. Transient loss of consciousness ('blackouts') in over 16S. clinical guideline 109 London, UK 2010 https://www.nice.org.uk/guidance/cg109/evidence (Accessed March 9, 2018).

5 , Shen W-K, Sheldon RS, et al, Writing Committee Members. 2017 ACC/AHA/HRS guideline for the evaluation and management of patients with syncope: a report of the American College of Cardiology/American heart association Task force on clinical practice guidelines and the heart rhythm Society. Heart Rhythm 2017; 14:e155-217.

6 Davis S, Westby M, Petkar S, et al. Tilt testing is more cost-effective than implantable loop recorder monitoring as a means of directing pacing therapy in people with recurrent episodes of suspected vasovagal syncope that affect their quality of life or present a high risk of injury. Heart 2013;99:805-10.

7 Davis S, Westby M, Pitcher D, et al. Implantable loop recorders are cost-effective when used to investigate transient loss of consciousness which is either suspected to be arrhythmic or remains unexplained. Europace 2012;14:402-9.

8 Providência R, Candeias R, Morais C, et al. Financial impact of adopting implantable loop recorder diagnostic for unexplained syncope compared with conventional diagnostic pathway in Portugal. BMC Cardiovasc Disord 2014:14:1-11.

9 Rogers J, Chang S, Quiroz ME, et al. The cost-effectiveness of detecting arrhythmia with implantable loop recorders in the United state of America. Value in Health 2014;17:A116.

10 Trikalinos TA, Siebert U, Lau J. Decision-analytic modeling to evaluate benefits and harms of medical tests: uses and limitations. Med Decis Making 2009;29:E22-9.

11 Neumann PJ, Ganiats TG, Russell LB, et al. Cost-effectiveness in health and medicine. Online: Oxford Scholarship Online, 2016.

12 Baugh CW, Liang L-J, Probst MA, et al. National cost savings from observation unit management of syncope. Acad Emerg Med 2015;22:934-41.

13 Orozco JJ, Shrivastav M, Vilendrer S. Cost-Effectiveness analysis of external looping recording compared to Holter monitoring for syncope in Colombia. Value in Health 2015;18:A44-5.

14 Symmetron Inc. Cost-effectiveness of reveal in the detection of arrhythmia: a USA perspective. data on file. Medtronic Inc., 2013. accessed.

15 Farwell DJ, Freemantle N, Sulke AN. Use of implantable loop recorders in the diagnosis and management of syncope. Eur Heart $J$ 2004;25:1257-63.

16 Centers for Disease Control and Prevention. United States life tables, 2014. Available: https://www.cdc.gov/nchs/products/life_tables.htm [Accessed April 10, 2018].

17 Burkowitz J, Merzenich C, Grassme K, et al. Insertable cardiac monitors in the diagnosis of syncope and the detection of atrial fibrillation: a systematic review and meta-analysis. Eur J Prev Cardiol 2016;23:1261-72.

18 Solbiati M, Casazza G, Dipaola F, et al. The diagnostic yield of implantable loop recorders in unexplained syncope: a systematic review and meta-analysis. Int J Cardiol 2017;231:170-6.

19 Bartoletti A, Fabiani P, Bagnoli L, et al. Physical injuries caused by a transient loss of consciousness: main clinical characteristics of patients and diagnostic contribution of carotid sinus massage. Eur Heart J 2008;29:618-24.

20 Brignole M, Sutton R, Menozzi C, et al. Early application of an implantable loop recorder allows effective specific therapy in patients with recurrent suspected neurally mediated syncope. Eur Heart J 2006;27:1085-92.
21 Antiarrhythmics versus Implantable Defibrillators (AVID) Investigators. A comparison of antiarrhythmic-drug therapy with implantable defibrillators in patients resuscitated from near-fatal ventricular arrhythmias. N Engl J Med 1997;337:1576-84.

22 Spector $\mathrm{P}$, Reynolds MR, Calkins $\mathrm{H}$, et al. Meta-analysis of ablation of atrial flutter and supraventricular tachycardia. Am J Cardiol 2009:104:671-7.

23 van Dijk N, Sprangers MA, Colman N, et al. Clinical factors associated with quality of life in patients with transient loss of consciousness. J Cardiovasc Electrophysiol 2006;17:998-1003.

24 Ara R, Brazier J. Deriving an algorithm to convert the eight mean SF-36 dimension scores into a mean EQ-5D preference-based score from published studies (where patient level data are not available). Value Health 2008;11:1131-43.

25 Barón-Esquivias G, Gómez S, Aguilera A, et al. Short-Term evolution of vasovagal syncope: influence on the quality of life. Int $J$ Cardiol 2005:102:315-9.

26 Romme JJCM, Reitsma JB, Go-Schön IK, et al. Prospective evaluation of non-pharmacological treatment in vasovagal syncope. Europace 2010;12:567-73.

27 Rose MS, Koshman M-L, Ritchie D, et al. The development and preliminary validation of a scale measuring the impact of syncope on quality of life. Europace 2009;11:1369-74.

28 Sheldon R, Rose S, Flanagan P, et al. Risk factors for syncope recurrence after a positive tilt-table test in patients with syncope. Circulation 1996:93:973-81.

29 Rodrigues IG, Lima MG, de Azevedo Barros MB, Barros M. Falls and health-related quality of life (SF-36) in elderly people-ISACAMP 2008. Health 2013;05:49-57.

30 Edvardsson N, Frykman V, van Mechelen R, et al. Use of an implantable loop recorder to increase the diagnostic yield in unexplained syncope: results from the PICTURE registry. Europace 2011;13:262-9.

31 Caro JJ, Briggs AH, Siebert U, et al. Modeling good research practices-overview: a report of the ISPOR-SMDM Modeling Good Research Practices Task Force-1. Value Health 2012:15:796-803.

32 Institute for Clinical and Economic Review (ICER). A guide to ICER's methods for health technology assessment, 2018. Available: http:// icer-review.org/wp-content/uploads/2018/08/ICER-HTA-Guide 082018.pdf [Accessed July 8, 2018].

33 National Institute for Health and Care Excellence (NICE). Guide to the methods of technology appraisal 2013, 2018. Available: www. nice.org.uk/process/pmg9 [Accessed July 8, 2019].

34 ClinicalTrials.gov. ClinicalTrials.gov Identifier: NCT02817906. ITI-007 for the Treatment of Agitation in Patients With Dementia, Including Alzheimer's Disease, 2018. Available: https://clinicaltrials.gov/ct2/ show/NCT02817906 [Accessed December 14, 2018].

35 Donateo P, Brignole M, Menozzi C, et al. Mechanism of syncope in patients with positive adenosine triphosphate tests. J Am Coll Cardiol 2003;41:93-8.

36 Frazier-Mills CG, Johnson LC, Xia Y, et al. Lower rates of syncope recurrence and less downstream diagnostic testing after Insertable cardiac monitor placement for syncope. American Cardiology Conference, New Orleans, USA, 2019.

37 Krahn AD, Klein GJ, Yee R, et al. Randomized assessment of syncope trial: conventional diagnostic testing versus a prolonged monitoring strategy. Circulation 2001;104:46-51.

38 Krahn AD, Klein GJ, Yee R, et al. Use of an extended monitoring strategy in patients with problematic syncope. Reveal Investigators. Circulation 1999;99:406-10.

39 Podoleanu C, DaCosta A, Defaye P, et al. Early use of an implantable loop recorder in syncope evaluation: a randomized study in the context of the French healthcare system (fresh study). Arch Cardiovasc Dis 2014;107:546-52.

40 Sulke N, Sugihara C, Hong P, et al. The benefit of a remotely monitored implantable loop recorder as a first line investigation in unexplained syncope: the EaSyAS II trial. Europace 2016;18:912-8.

41 Ara R, Wailoo A. Using health state utility values in models exploring the cost-effectiveness of health technologies. Value Health 2012;15:971-4

42 Mant J, Fitzmaurice DA, Hobbs FDR, et al. Accuracy of diagnosing atrial fibrillation on electrocardiogram by primary care practitioners and interpretative diagnostic software: analysis of data from screening for atrial fibrillation in the elderly (SAFE) trial. $B M J$ 2007;335:380.

43 Farwell DJ, Freemantle N, Sulke N. The clinical impact of implantable loop recorders in patients with syncope. Eur Heart J 2006;27:351-6.

44 Musat Det al. Understanding automatic connectivity limitations in patients undergoing long-term ECG monitoring with an implantable cardiac monitor. Heart Rhythm Sessions 2017. 